\title{
Conservación
}

\section{Fragmentación progresiva y pérdida de hábitat de bosques naturales en uno de los hotspot mundiales de biodiversidad}

\author{
Progressive fragmentation and loss of natural forests habitat in one of the global biodiversity hotspot \\ Samuel Otavo ${ }^{\text {a,* }}$ y Cristian Echeverría ${ }^{\text {a,b }}$ \\ ${ }^{a}$ Laboratorio de Ecología del Paisaje, Facultad de Ciencias Forestales, Universidad de Concepción, Casilla 160-C - Correo 3, Concepción, Chile \\ ${ }^{\mathrm{b}}$ Núcleo Milenio Centro para el Impacto Socioeconómico de las Políticas Ambientales (CESIEP), Santiago, Chile
}

Recibido el 15 de diciembre de 2016; aceptado el 17 de mayo de 2017

Disponible en Internet el 6 de diciembre de 2017

\begin{abstract}
Resumen
El conocimiento del estado de fragmentación y transformación de un paisaje boscoso es crucial para una adecuada planificación y conservación de la biodiversidad. En Chile se encuentra uno de los hotspots mundiales de biodiversidad; dentro de este, se encuentra la cordillera de Nahuelbuta, la cual es considerada como un área de alto valor de biodiversidad y de intensa presión antrópica. A pesar de ello, no se cuenta con información precisa sobre el grado de transformación de su paisaje y su estado de conservación. El objetivo de este trabajo fue evaluar el estado del paisaje y los cambios espacio-temporales de los bosques nativos en dicha cordillera. A partir de imágenes Landsat de los años 1986 y 2011 , se generaron mapas temáticos de usos del suelo. Se observó una pérdida de bosque nativo del $33 \%$ en 25 años asociada principalmente a la sustitución por plantaciones forestales. Los cambios en los patrones espaciales de las coberturas y usos del suelo revelan una profunda transformación del paisaje y una fragmentación avanzada de los bosques nativos. Se discute como estos patrones de cambio amenazan la persistencia de diversas especies endémicas con alto riesgo de extinción. De continuar estos procesos antrópicos, estas especies podrían enfrentar un aumento en su riesgo de extinción.
\end{abstract}

(C) 2017 Universidad Nacional Autónoma de México, Instituto de Biología. Este es un artículo Open Access bajo la licencia CC BY-NC-ND (http://creativecommons.org/licenses/by-nc-nd/4.0/).

Palabras clave: Deforestación; Cordillera de Nahuelbuta; Diversidad; Endemismo; Conservación a escala de paisaje; Efecto de las plantaciones exóticas

\begin{abstract}
The understanding of the degree of forest fragmentation and landscape transformation is crucial for landscape sustainability and biodiversity conservation. Within the Chilean hotspot the Nahuelbuta Mountain can be found, which is considered as a high biodiversity value area under an intense human pressure. However, there is no information on the degree of transformation of the landscape and the conservation status of its natural forest ecosystems. The aim of this study was to evaluate the state of the landscape and the spatiotemporal changes in native forests in Nahuelbuta. Through the use of Landsat imagery acquired for 1986 and 2011, land-cover and use maps were generated. Results revealed a loss of $33 \%$ of native forest in 25 years, mainly associated with the conversion to forest plantations. Changes in spatial patterns of land-cover and uses revealed a profound transformation of the study landscape and an advanced fragmentation of native forests. We discuss how these changes threaten the persistence of different endemic species at high risk of extinction. If the current trend of spatial pattern changes continues, it is probable that these species might face an increase in their extinction risk.
\end{abstract}

(C) 2017 Universidad Nacional Autónoma de México, Instituto de Biología. This is an open access article under the CC BY-NC-ND license (http://creativecommons.org/licenses/by-nc-nd/4.0/).

Keywords: Deforestation; Nahuelbuta Mountain; Diversity; Endemism; Landscape-scale conservation; Effect of exotic plantations

\footnotetext{
* Autor para correspondencia.

Correo electrónico: samuelotavo@udec.cl (S. Otavo).

La revisión por pares es responsabilidad de la Universidad Nacional Autónoma de México.
} 


\section{Introducción}

El estado de transformación de un paisaje boscoso se caracteriza por el grado de destrucción y modificación del hábitat, la tasa de deforestación, los patrones de fragmentación de bosques naturales y los cambios en la matriz (Echeverría, Newton, Nahuelhual, Coomes y Rey-Benayas, 2012; McIntyre y Hobbs, 1999). En este sentido, se pueden encontrar paisajes poco modificados, con baja tasa de deforestación, y áreas extensas y bien conectadas de bosques nativos (Echeverría et al., 2012), o paisajes altamente fragmentados y modificados, donde la cobertura boscosa ocupa una reducida área del paisaje, con fragmentos de hábitat dispersos, aislados y sin conectividad, y una matriz compuesta por usos antrópicos (Echeverría et al., 2006; Steininger et al., 2001). El conocimiento del estado de transformación de un paisaje es un antecedente crucial para una adecuada planificación, manejo y restauración del paisaje, ya que indican el grado de integridad o funcionalidad de estos y los principales atributos que requieren ser mantenidos o mejorados para la conservación de la biodiversidad y el bienestar humano (Liu y Taylor, 2002). Estos antecedentes son especialmente importantes en paisajes con alta diversidad y endemismos de especies de flora y fauna, pero con pérdidas substanciales de hábitats boscosos (Steininger et al., 2001).

La fragmentación de bosques naturales es una de las principales causas de pérdida de biodiversidad en el mundo (Turner, 1996). Los principales efectos de la fragmentación a nivel de paisaje son la reducción del tamaño y calidad del hábitat boscoso, el aumento de borde y número de parches, y la pérdida de conectividad (Forman y Godron, 1986; Lindenmayer y Fischer, 2006). El borde de un parche en el paisaje es definido como la zona de transición entre hábitats, y su percepción varía dependiendo del observador, organismo o variable estudiada (López-Barrera, 2004). Los fragmentos boscosos pueden llegar a tener bordes suaves o abruptos, dependiendo del grado de contraste entre hábitats, lo cual desempeña un papel crítico en la capacidad de las especies de adaptarse y moverse dentro de hábitats perturbados, la capacidad de recuperación del bosque y la penetración de los efectos de borde que originan un deterioro en la calidad del hábitat en regresión (Bennett, 1999; Lindenmayer y Fischer, 2006; Peyras, Vespa, Bellocq y Zurita, 2013). La reducción del tamaño de los fragmentos boscosos genera cambios en la composición y estructura a nivel de comunidades (Echeverría, Newton, Lara, Rey-Benayas y Coomes, 2007; Tabarelli, Mantovani y Peres, 1999), modificando las dinámicas poblacionales y diversos procesos ecológicos (Laurance et al., 2002; Wiegand, Revilla y Moloney, 2005) que, junto al aislamiento y la disminución de la conectividad funcional, tienen efectos negativos sobre la persistencia de poblaciones de especies (Crooks, Burdett, Theobald, Rondinini y Boitani, 2011; Lindenmayer y Fischer, 2006). Se ha constatado que la continua fragmentación y pérdida de bosques tropicales y templados han afectado la riqueza y estructura del bosque, la abundancia y diversidad de aves, el ensamble de comunidades de insectos y la persistencia de poblaciones de mamíferos, entre otros (Barbosa y Marquet, 2002; Castelletta, Thiollay y Sodhi, 2005; De Angelo, Paviolo y di Bitetti, 2011; Echeverría et al., 2007; Stratford y Stouffer, 2015).
Los hotspot son regiones con prioridad mundial para la conservación por su alto grado de endemismo y fuerte impacto antrópico; cifras alarmantes señalan que más del $85 \%$ del hábitat original presente en los hotspot ha sido destruido (Mittermeier, Turner, Larsen, Brooks y Gascon, 2011). En la actualidad se han definido 35 hotspots, entre los cuales el hotspot «Chilean winter rainfall-Valdivian forests» se caracteriza por albergar un total de 3,893 especies de plantas vasculares nativas, el 50\% de ellas endémicas del propio hotspot y contener más de la mitad de los bosques templados del hemisferio sur (Mittermeier et al., 2011). Uno de los paisajes más singulares, menos protegidos y más alterados del hotspot chileno es aquel presente en la cordillera de Nahuelbuta («cordillera», de aquí en adelante) (Smith-Ramírez, 2004). Esta zona es considerada por algunos científicos como un área de alto valor de conservación y reserva mundial de la biodiversidad, por sus altos niveles de diversidad de especies y endemismo, y características físicas que favorecieron la persistencia de algunas especies, incluso durante la última glaciación (Smith-Ramírez, 2004; Wolodarsky-Franke y Díaz, 2011). La alta riqueza de especies y endemismo de esta área se atribuye al hecho que los bosques deciduos y matorrales característicos de la zona mediterránea del norte de Chile convergen con la vegetación siempreverde valdiviana del sur de Chile, formando un singular ecosistema ecotonal y por poseer especies remanentes desde el Mezosoico de origen Gondwanico, y especies del Terciario de origen tropical (Smith-Ramírez, 2004).

A pesar de su alto valor para la conservación de la biodiversidad, diversos procesos antrópicos, tales como la sustitución de bosque nativo por plantaciones de especies exóticas, incendios forestales, extracción de leña, agricultura intensiva y sobreexplotación de especies nativas, han sido asociados a la degradación y pérdida de los bosques naturales de esta «cordillera» (SmithRamírez, 2004; Wolodarsky-Franke y Díaz, 2011); lo cual ha afectado diferentes especies de flora y fauna endémicas de Chile y presentes en la «cordillera», que se encuentran amenazadas y en riesgo de extinción, como los árboles, queule (Gomortega keule (Mol.) Baillon) y pitao (Pitavia punctata (R. et P.) Mol.), los anfibios, ranita de Darwin (Rhinoderma darwini (Duméril et Bibron, 1841)) y sapo de Contulmo (Eupsohus contulmoensis (Ortiz, Ibarra y Formas, 1989)), entre los mamíferos, el zorro de Darwin (Lycalopex fulvipes (Martin, 1837)) y el marsupial (Dromiciops gliroides (Thomas, 1894)) (Hechenleitner et al., 2005; Wolodarsky-Franke y Díaz, 2011), entre muchos otros. Si bien existen estudios puntuales de la transformación de los paisajes costeros de Chile (Miranda, Altamirano, Cayuela, Lara y González, 2017), no existen estudios espacio-temporales sobre el estado de transformación de la «cordillera», y en particular, de los cambios en los patrones espaciales de sus bosques nativos. Debido a ello, no se cuenta con información básica para planificar acciones o estrategias de conservación y restauración a escala de paisaje.

El objetivo de este trabajo es evaluar el estado del paisaje y los cambios espacio-temporales de los bosques nativos en la «cordillera», y a partir de estos resultados, discutir las implicaciones que tienen tales cambios, en la persistencia de varias especies de flora y fauna amenazadas de la «cordillera», dependientes de hábitats de bosque nativo. Es posible esperar que 
debido a la constante pérdida y sustitución de bosque nativo por plantaciones forestales de especies exóticas y habilitación de áreas para agricultura, la «cordillera» se encuentre en un estado avanzado de transformación y fragmentación de los bosques naturales.

\section{Materiales y métodos}

La «cordillera» se localiza entre el río Biobío y el río Imperial, en las regiones administrativas del Biobío y La Araucanía, respectivamente, y se extiende por $200 \mathrm{~km}$ en sentido norte sur, alcanzando una altitud máxima de $1,530 \mathrm{~m}$ snm (fig. 1). Las precipitaciones se concentran en un $80 \%$ en los meses de otoño e invierno, aumentando en 2 gradientes, uno latitudinal (norte sur) y otro longitudinal (oeste este), con frecuentes nevadas por encima de los 1,000 m snm en los meses de invierno (Di Castri y Hajek, 1976).

Debido a que no existe ningún estudio que defina con precisión los límites de la «cordillera», en el presente trabajo se establecieron límites con base a criterios geológicos, pedológicos, vegetacionales y altimétricos. El criterio geológico considera la formación de la «cordillera» en el Paleozoico superior, siendo mucho más antigua que la cordillera de Los Andes, que data de fines del Terciario (Mardones, 2005). Se excluyeron en esta delimitación a secuencias sedimentarias marinas plataformales y depósitos piroclásticos asociados a calderas de colapso (Mardones, 2005; SERNAGEOMIN, 2003).
La pedología de la «cordillera» corresponde en su mayoría a suelos ultisoles de la serie Asociación Nahuelbuta generados a partir de rocas metamórficas, de textura arcillosa, franco-arcillolimosa y arcillo-limosa, profundos y generalmente de topografía escarpada (CIREN, 1999). La redelimitación a partir de formaciones vegetacionales consistió en suavizar y cerrar los bordes del producto obtenido de las anteriores redelimitaciones, para lo cual se usó la clasificación de pisos de vegetaciones propuesto por Luebert y Pliscoff (2006). El límite altimétrico se basó en la cota de $200 \mathrm{~m} \mathrm{snm}$, debido a su congruencia espacial con los demás criterios analizados.

Para generar mapas temáticos de los usos del suelo, se utilizaron imágenes satelitales Landsat de primavera y verano de 1986 (TM) y 2011 (ETM+), con una nubosidad inferior del 10\%. Para facilitar su procesamiento y llevar a cabo la comparación cuantitativa de las coberturas de uso del suelo, las imágenes se proyectaron a una resolución espacial de $30 \times 30 \mathrm{~m} /$ píxel y, posteriormente, fueron corregidas geométrica, atmosférica y topográficamente (Chander, Markham y Helder, 2009). Por medio de la metodología del factor $\mathrm{C}$, se eliminaron las sombras proyectadas por la topografía del lugar (Reese y Olsson, 2011). Para aumentar la exactitud de la clasificación fueron utilizados los índices de vegetación NDVI, SR, SAVI y LSWI (Huete, 1988; Rouse, Haas, Schell y Deering, 1974; Tucker, 1979).

Se realizó una clasificación supervisada para cada imagen utilizando el método estadístico de máxima verosimilitud y puntos de entrenamiento, los cuales representan los patrones de los

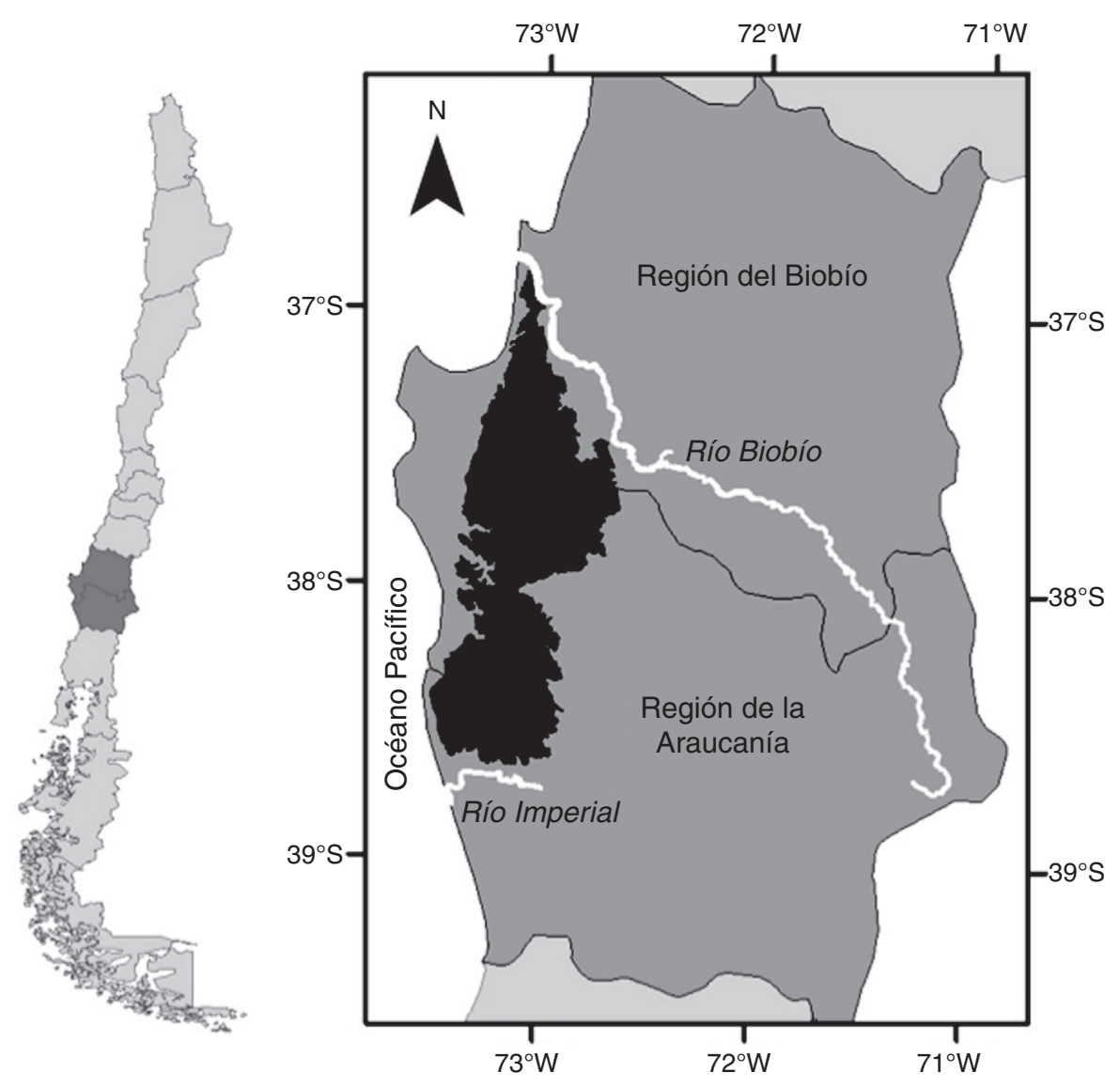

Figura 1. Localización de la cordillera de Nahuelbuta entre el río Biobío y el río Imperial en las regiones administrativas del Biobío y La Araucanía, Chile. 
Tabla 1

Métricas del paisaje usadas para el análisis de patrones espaciales de los bosques nativos de la cordillera de Nahuelbuta (McGarigal et al., 2013).

\begin{tabular}{|c|c|c|c|c|}
\hline Clase & Métrica & Descripción & Unidad de medida & Rango de variación \\
\hline \multirow[t]{4}{*}{$\begin{array}{l}\text { Área, densidad } \\
\text { y borde }\end{array}$} & Área del parche & $\begin{array}{l}\text { Área de cada parche de bosque nativo en } \\
\text { el paisaje }\end{array}$ & Hectáreas & 0 y sin límite \\
\hline & $\begin{array}{l}\text { Índice del parche más } \\
\text { grande }\end{array}$ & $\begin{array}{l}\text { Porcentaje del área del parche de bosque } \\
\text { nativo más grande respecto } \\
\text { al área total del paisaje }\end{array}$ & Porcentaje & $0<\mathrm{LPI} \leqq 100$ \\
\hline & Densidad de parches & $\begin{array}{l}\text { Número de parches de bosque nativo por } \\
\text { cada } 100 \text { hectáreas }\end{array}$ & Numérico & $\mathrm{PD}>0$ \\
\hline & Densidad de borde & $\begin{array}{l}\text { Densidad de la longitud de borde de los } \\
\text { parches de bosque nativo en el paisaje }\end{array}$ & Metros por hectárea & $\mathrm{ED} \geq 0$, sin límite \\
\hline Forma & $\begin{array}{l}\text { Relación media } \\
\text { perímetro-área }\end{array}$ & Relación entre el perímetro y el área & Ninguna & PARA $>0$ y sin límite \\
\hline Área núcleo & Área núcleo media & $\begin{array}{l}\text { Área núcleo del bosque nativo, } \\
\text { especificada por la profundidad de borde } \\
\text { según vecindad del parche con otros usos } \\
\text { del suelo }\end{array}$ & Hectáreas & $\mathrm{CORE} \geq 0$ y sin límite \\
\hline $\begin{array}{l}\text { Aislamiento } \\
\text { Proximidad }\end{array}$ & $\begin{array}{l}\text { Distancia media al } \\
\text { vecino más cercano }\end{array}$ & $\begin{array}{l}\text { Proximidad de los parches de bosque } \\
\text { nativo, basado en la distancia media } \\
\text { ponderada }\end{array}$ & Metros & $\mathrm{ENN}>0 \mathrm{y}$ sin límite \\
\hline Contraste & $\begin{array}{l}\text { Contraste de borde } \\
\text { medio ponderado } \\
\text { por el área }\end{array}$ & $\begin{array}{l}\text { Grado de contraste del bosque nativo } \\
\text { respecto a su densidad. El contraste } \\
\text { representa la magnitud de la diferencia } \\
\text { entre el Bosque nativo } \\
\text { y los demás usos del suelo }\end{array}$ & Porcentaje & $0 \leqq \mathrm{ECON} \leqq 100$ \\
\hline $\begin{array}{l}\text { Contagio } \\
\text { Interspersion }\end{array}$ & Índice de agregación & $\begin{array}{l}\text { Desviación proporcional de adyacencias } \\
\text { que implica la clase de bosque nativo a } \\
\text { partir de la esperada para una } \\
\text { distribución espacialmente aleatoria }\end{array}$ & Porcentaje & -1 _CLUMPY _ 1 \\
\hline
\end{tabular}

tipos de cobertura del suelo. Un total de 300 puntos de entrenamiento fueron tomados en diferentes campañas en terreno para la clasificación de las imágenes más recientes. Mientras que para las imágenes de 1986, se utilizaron mapas de la cobertura del suelo generados por estudios previos (Conaf et al., 1999; Lara, Araya, Capella, Fierro y Cavieres, 1989) y consulta local de coberturas de uso del suelo que no mostraron cambio de uso en los últimos 20 años. La precisión en la clasificación de las imágenes se calculó por medio de matrices de confusión, a partir de un rango de entre 60 y 90 puntos de validación tomados en terreno y fotografías aéreas recientes, por cada tipo de cobertura, de cada imagen satelital. Se realizó un filtro por clasificación a cada una de las clases de coberturas presentes con el objetivo de excluir parches con menos de 4 pixeles.

Las clases de coberturas o usos del suelo del paisaje de la «cordillera» identificados a partir de cada imagen fueron: 1) bosque nativo primario (bosques antiguos originados por sucesión natural); 2) bosque nativo secundario (regeneración de bosques posterior a un disturbio); 3) bosque achaparrado; 4) matorral arborescente; 5) matorral; 6) plantaciones forestales exóticas (plantaciones comerciales); 7) suelo agrícola (cultivos agrícolas, praderas y zonas de uso ganadero), y 8) otros usos y coberturas (cuerpos de agua, urbano, nubes y sombras).

La transición de las coberturas de bosques nativos a otros usos del suelo fueron analizados mediante el módulo Análisis de Cambio de la extensión Land Change Modeler del software IDRISI (IDRISI, 2012). En el caso de cambios en bosques nativos, se empleó la siguiente fórmula para determinar la tasa de deforestación anual:

$P=\left[\left(\frac{A_{2}}{A_{1}}\right)^{\left(\frac{1}{\left(t_{2}-t_{1}\right)}\right)}-1\right] \times 100$

donde $A_{1}$ y $A_{2}$ son el área de bosque nativo en el tiempo $t_{1}$ y $t_{2}$, respectivamente, y $P$ es el porcentaje de pérdida por año (Newton, 2007).

El estado actual del paisaje fue analizado con base en los modelos de cambio del paisaje propuestos por McIntyre y Hobbs (1999) y Echeverría et al. (2012). Específicamente, se emplearon las siguientes variables: pérdida y fragmentación de bosques nativos, tasa de deforestación, proceso dominante del paisaje, y cambios en la matriz durante el periodo estudiado. Por otra parte, la transformación del paisaje se midió como porcentaje de hábitat remanente en términos de bosques naturales (McIntyre y Hobbs, 1999).

Para el análisis de los patrones espaciales de la cobertura boscosa nativa entre 1986 y 2011, se usó el software FRAGSTATS (McGarigal, Cushman, Neel y Ene, 2013). La elección del conjunto de métricas a utilizar se basó en la revisión de diversos estudios sobre métricas del paisaje que expresaran en mejor forma la configuración espacial de paisajes reales, y además fueran representativas de componentes esenciales de la estructura del paisaje como son: la calidad de parche, el borde del parche, el contexto del parche en el paisaje y la conectividad del parche (tabla 1) (Cushman, McGarigal y Neel, 2008; Liu y Taylor, 2002; Mas, Gao y Pacheco, 2010; Peng et al., 2010; Zeng y Wu, 2005) 
Para el cálculo del índice de contraste se asignaron pesos de contraste entre los bordes de parches de bosques nativos y los demás tipos de coberturas presentes en el área de estudio. El peso de contraste de los bordes se determinó con base en variables de composición y estructura de la vegetación, medidas en 13 parcelas de muestreo de $20 \times 10 \mathrm{~m}$. Para el cálculo de área núcleo se emplearon distancias perpendiculares desde el borde hacia el centro del parche, la cual correspondió a la zona de efecto borde entre el bosque nativo y los demás tipos de coberturas. La distancia asignada fue con base en los trabajos realizados por Laurance et al. (2002), López-Barrera (2004) y Lindenmayer y Fischer (2006), considerando que el grado de contraste entre hábitats (bordes suaves o abruptos) expresa la magnitud y distancia de respuestas primarias y secundarias de la estructura, composición y procesos del hábitat ante los efectos de borde.

\section{Resultados}

Clasificación de imágenes. La precisión de la clasificación de 1986 fue del 87\%, siendo la clase plantaciones exóticas la menos precisa $(81 \%)$, confundiéndose con coberturas de bosques nativos primarios. Los bosques nativos primarios para 1986 tuvieron una precisión del 88\%, mientras que los bosques nativos secundarios y achaparrados tuvieron una precisión del 86 y 87\%, respectivamente. Para el año 2011 la precisión de clasificación se estimó en un $85 \%$, siendo la clase matorral arborescente la menos precisa $(77 \%)$. Las plantaciones forestales tuvieron un

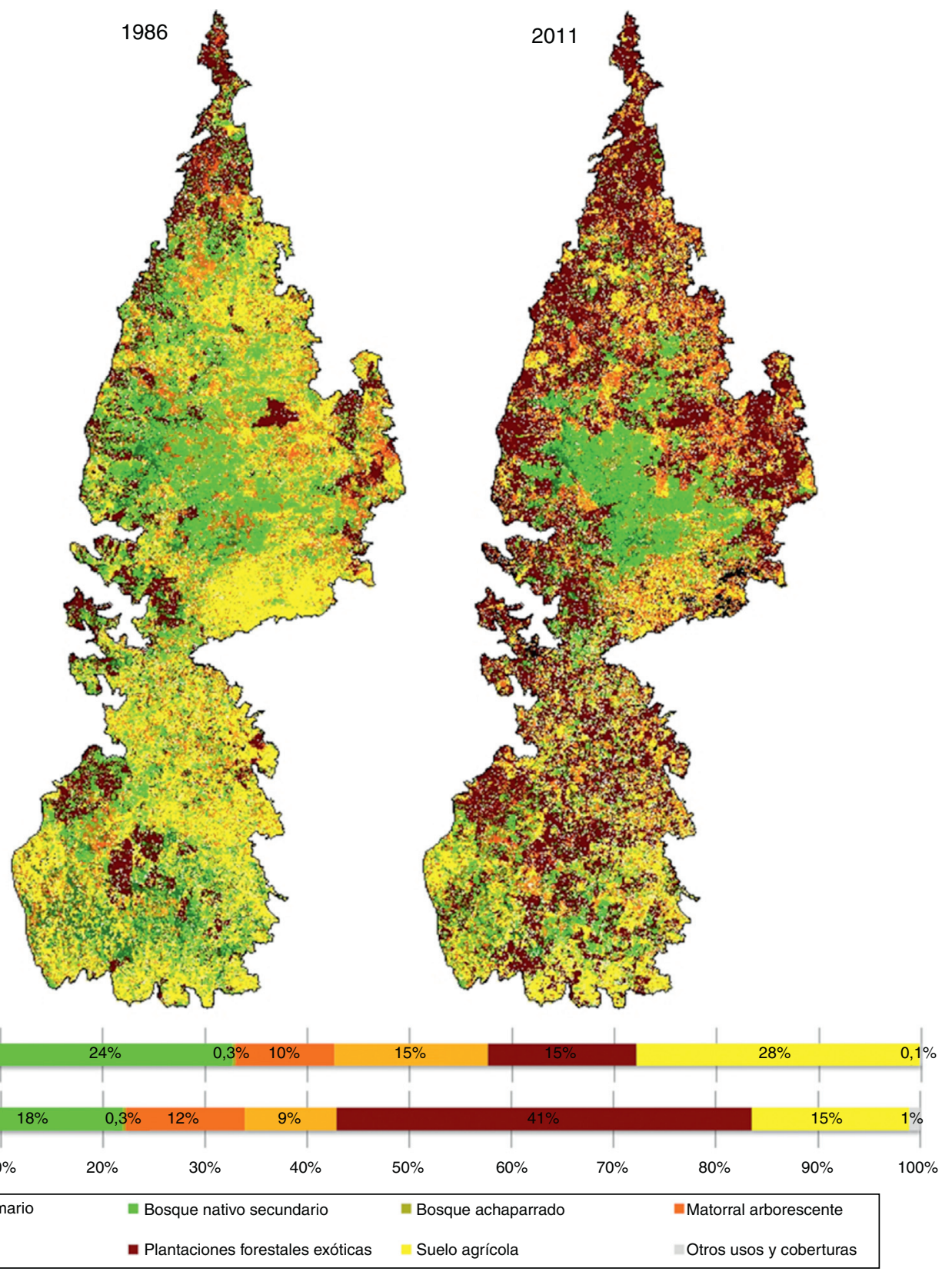

Figura 2. Variación espacio-temporal y porcentaje del área ocupada por los tipos de cobertura del suelo en 1986 y 2011 en la cordillera de Nahuelbuta. 

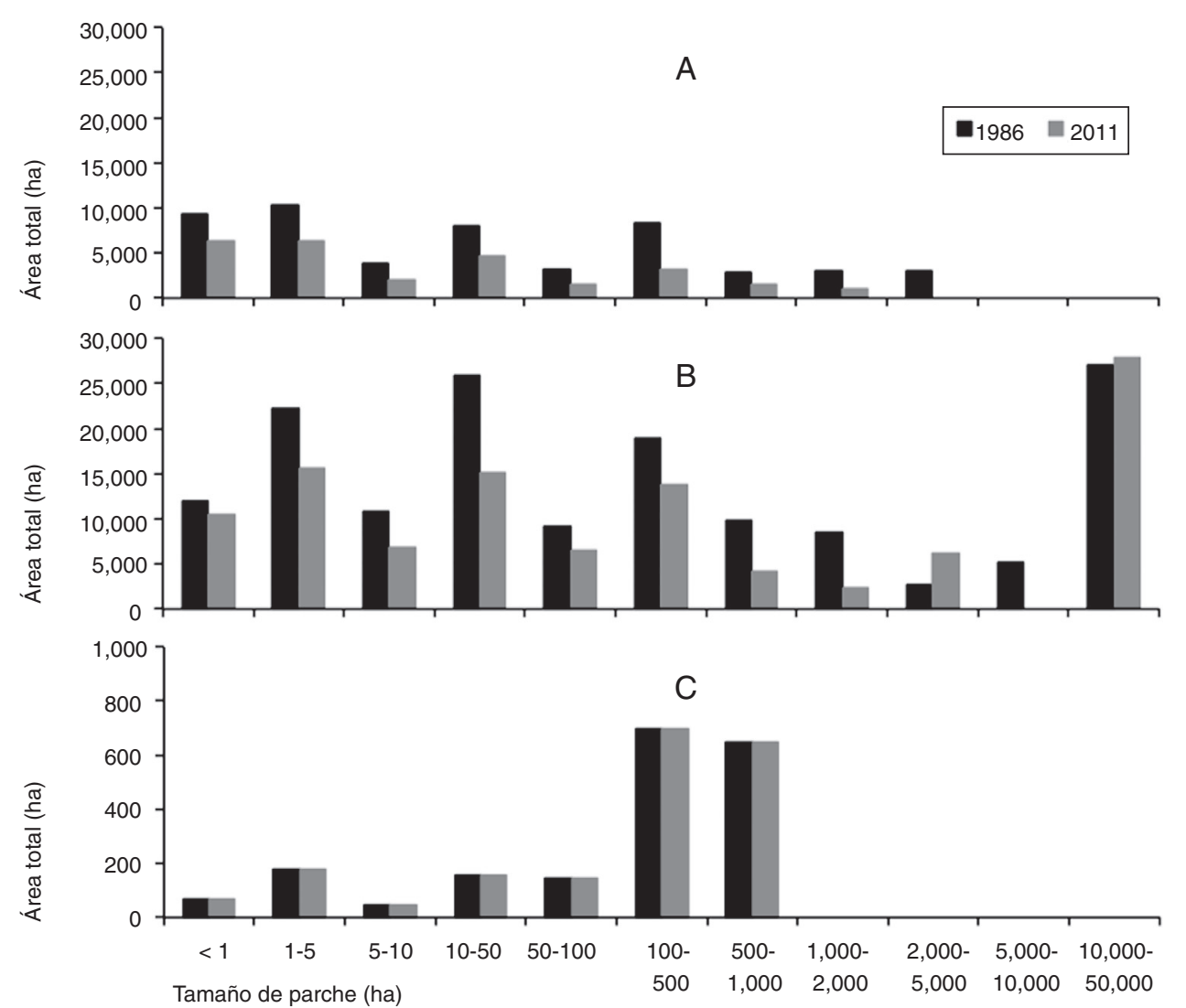

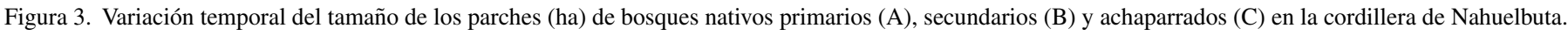

$78 \%$ de precisión, mientras que bosques nativos primarios obtuvieron un $86 \%$, bosques nativos secundarios un $85 \%$ y bosques achaparrados un $88 \%$.

Pérdida de cobertura boscosa. La «cordillera» posee un área aproximada a 620,000 ha, de las cuales 206,130 ha $\pm 26,8$ estaban constituidas por bosques nativos en 1986, disminuyendo a 137,700 ha $\pm 19,3$ al año 2011 (fig. 2). Es decir, en 25 años la cobertura boscosa disminuyó un $33.2 \%$ con una tasa de deforestación del $1.6 \%$ año $^{-1}$. La pérdida neta fue más intensa en bosques nativos primarios que en bosques nativos secundarios, mientras que los bosques achaparrados no presentaron pérdida. En 1986 los bosques nativos primarios ocupaban tan solo un $8 \%$ del total del paisaje $(52,019 \mathrm{ha})$, reduciendo su área a la mitad en el 2011, con una tasa de deforestación del $2.6 \%$ año $^{-1}$ (fig. 2). En comparación, los bosques nativos secundarios presentaron una pérdida neta de 43,000 ha, lo que representa un $28 \%$ del área original en 1986 (152,200 ha), con una tasa de deforestación del $1.3 \%$ año ${ }^{-1}$ (fig. 2).

En cuanto a las transiciones de bosques nativos primarios, un $68 \%$ de la pérdida neta ocurrió por la sustitución a plantaciones de especies exóticas y en menor proporción por degradación a bosques nativos secundarios (11\%) y matorrales arborescentes (12\%). Por otra parte, un $95 \%$ de los cambios de cobertura de bosques nativos secundarios correspondió a la sustitución por plantaciones de especies exóticas. En este mismo periodo de estudio (1986-2011), las plantaciones de especies exóticas se incrementaron casi un 150\% aumentando de 90,750 ha en 1986 a 251,250 ha en el 2011, con una tasa de forestación del $4.2 \%$ año ${ }^{-1}$, siendo la cobertura predominante el 2011 (fig. 2).

Análisis de los patrones espaciales de la cobertura boscosa nativa. Se observaron cambios considerables en la distribución del tamaño de los parches de la cobertura boscosa durante el periodo 1986-2011 (fig. 3). En 1986, el 61\% del área de bosque nativo primario se encontraba en parches inferiores a $50 \mathrm{ha}$, el $22 \%$ en parches de 50 a 500 ha y un $17 \%$ distribuido en 7 parches mayores de 500 ha (fig. 3). Posteriormente, en el 2011 , de las 26,600 ha de bosque nativo primario existente, el $72 \%$ estaba distribuido en parches inferiores a 50 ha, un $18 \%$ en parches de 50 a 500 ha y tan solo un $10 \%$ se distribuía en 3 parches de tamaño mayor que 500 ha (fig. 3). En el año 2011, se observó un único gran parche de bosque nativo primario de 1,120 ha. De las 25,420 ha deforestadas de bosque nativo primario, el $49 \%$ ocurrió en parches de área inferior a 50 ha.

En relación con el bosque nativo secundario, aproximadamente el 50\% se encontraba en parches de área inferior a 50 ha, persistiendo en el tiempo un único gran fragmento, que representaba el $4.3 \%$ del área total del paisaje en 1986 y el $4.5 \%$ en el 2011 (fig. 3). En el periodo de estudio, el bosque nativo secundario presentó una regeneración aproximada de 5,680 ha, de las cuales un $82 \%$ ocurrió en parches de 2,000 a 5,000 ha. No obstante, de las 43,000 ha deforestadas de bosque nativo secundario, el 53\% de la pérdida ocurrió en parches con tamaño inferior a las 50 ha. 
Tabla 2

Cambios en los índices de patrones del paisaje en la cordillera de Nahuelbuta a nivel de clase entre 1986 y 2011.

\begin{tabular}{|c|c|c|c|c|c|c|c|c|c|}
\hline \multirow[t]{2}{*}{ Tipo de cobertura boscosa } & \multirow{2}{*}{$\begin{array}{l}\text { Clase } \\
\text { Métrica }\end{array}$} & \multirow{2}{*}{$\begin{array}{l}\text { Contraste } \\
\text { Contraste de } \\
\text { borde medio } \\
\text { ponderado } \\
\text { por el área }\end{array}$} & \multirow{2}{*}{$\begin{array}{l}\text { Forma } \\
\text { Relación } \\
\text { media } \\
\text { perímetro- } \\
\text { área }\end{array}$} & \multicolumn{3}{|c|}{ Área, densidad y borde } & \multirow{2}{*}{$\begin{array}{l}\text { Aislamiento } \\
\text { Proximidad } \\
\text { Distancia } \\
\text { media al } \\
\text { vecino más } \\
\text { cercano }\end{array}$} & \multirow{2}{*}{$\begin{array}{l}\text { Contagio } \\
\text { Interspersion } \\
\text { Índice de } \\
\text { agregación }\end{array}$} & \multirow{2}{*}{$\begin{array}{l}\text { Área núcleo } \\
\begin{array}{l}\text { Área núcleo } \\
\text { media }\end{array}\end{array}$} \\
\hline & & & & $\begin{array}{l}\text { Densidad } \\
\text { de parches }\end{array}$ & $\begin{array}{l}\text { Densidad } \\
\text { de borde }\end{array}$ & $\begin{array}{l}\text { Índice del } \\
\text { parche más } \\
\text { grande }\end{array}$ & & & \\
\hline \multirow[t]{2}{*}{ Bosque nativo primario } & 1986 & 44.72 & 776.46 & 3.73 & 30.14 & 0.49 & 133.99 & 0.63 & 0.79 \\
\hline & 2011 & 42.16 & 817.77 & 2.51 & 19.82 & 0.18 & 154.67 & 0.57 & 0.76 \\
\hline & 2011 & 56.70 & 785.56 & 4.62 & 55.54 & 4.52 & 105.09 & 0.62 & 1.06 \\
\hline \multirow[t]{2}{*}{ Bosque achaparrado } & 1986 & 38.20 & 654.97 & 0.04 & 0.65 & 0.10 & 113.14 & 0.79 & 4.09 \\
\hline & 2011 & 29.03 & 654.97 & 0.04 & 0.61 & 0.10 & 113.14 & 0.79 & 4.64 \\
\hline
\end{tabular}

De 1986 a 2011 se observó un incremento en la complejidad de la forma de los fragmentos de bosque nativo primario y secundario (tabla 2). Por su parte, el grado de contraste de hábitat fue mayor en bosque nativo primario que en secundario, con una leve tendencia a decrecer en el tiempo (tabla 2). Los bosques achaparrados presentaron el menor grado de contraste en el periodo de estudio $(1986=38 \%, 2011=29 \%)$ (tabla 2$)$.

En 1986 la densidad de parches de bosque nativo primario y secundario fue de 3.73 y 5.58 , respectivamente (tabla 2). En el 2011, este índice se redujo a 2.51 fragmentos para el bosque nativo primario y 4.62 fragmentos para el bosque nativo secundario (tabla 2). Igualmente, la densidad de borde disminuyó de 1986 a 2011 para los 3 tipos de bosque, presentándose la mayor reducción de borde en bosque nativo secundario (tabla 2).

Se observó un incremento de la distancia entre los parches de bosque nativo primario y secundario (tabla 2). El índice de agregación de bosque nativo primario disminuyó, mientras que para bosque nativo secundario aumentó (tabla 2). No obstante, no se observó una variación considerable del índice de agregación de los fragmentos boscosos en el paisaje. Por su parte, los bosques achaparrados no presentaron cambios en la distancia media entre fragmentos, y en su índice de agregación (tabla 2).

El área núcleo de bosque nativo primario tuvo una pérdida del $4 \%$, lo cual concuerda con la disminución en un $63 \%$ del área del parche más grande para este tipo de bosque (tabla 2). En cambio, el bosque nativo secundario y el bosque achaparrado incrementaron en un $20 \%$ y $12 \%$ el área núcleo, respectivamente (tabla 2).

\section{Discusión}

Pérdida cobertura boscosa. El presente estudio reveló una pérdida total de bosque nativo del $33.2 \%$ a una tasa de deforestación del $1.6 \%$ año $^{-1}$ entre 1986 y 2011 en un paisaje de alto valor de conservación, inserto en un hotspot de biodiversidad. Esta pérdida fue más intensa en bosques nativos primaros (49\%) que en bosques nativos secundarios (28\%), y ocurrió principalmente por la sustitución a plantaciones forestales exóticas. Una pérdida de bosque nativo similar a la del presente estudio también fue observada en el hotspot chileno, entre las regiones del Biobío y
La Araucanía en el periodo 1979-2000, donde la pérdida neta boscosa fue del $28.2 \%$ a una tasa de deforestación del $1.6 \%$ año $^{-1}$ (Aguayo, Pauchard, Azócar y Parra, 2009). De este porcentaje de pérdida, el $71.7 \%$ ocurrió por sustitución de bosque a plantaciones forestales exóticas (Aguayo et al., 2009). Por otra parte, en el paisaje costero chileno de la región del Maule, entre 1975 y 2000 se estimó una pérdida de bosques nativos de un $67 \%$, el doble de lo registrado en el presente estudio, a una tasa de deforestación del $4.5 \%$ año $^{-1}$, como consecuencia principal de la sustitución por plantaciones forestales exóticas (Echeverría et al., 2006). Esto refleja la constante pérdida de bosque nativo por sustitución a plantaciones forestales exóticas y la actual ausencia de grandes extensiones de bosques nativos costeros en paisajes del hotspot chileno.

Pérdidas de hábitat boscosos en hotspot de biodiversidad también han sido reportadas en otros zonas del mundo para las últimas décadas. Por ejemplo, en el hotspot de la provincia de Limón en Costa Rica, la deforestación a 1997 fue de 54,830 ha, equivalente al $8.4 \%$ de la cubierta forestal existente en 1986 (Van Laake y Sánchez-Azofeifa, 2004). Porcentajes superiores de deforestación a lo estimado en la «cordillera» se observaron en el hotspot de las montañas del Arco Oriental de Tanzania, donde los bosques siempreverdes y sabanas arboladas naturales desaparecieron en un 48\% entre 1975 y 2000 (Green et al., 2013), y en el hotspot del Ecuador, ubicado en las provincias de Loja y Zamora Chinchipe, donde la pérdida de cobertura boscosa fue del 46\% entre 1976 y 2008 (Tapia-Armijos, Homeier, Espinosa, Leuschner y de la Cruz, 2015).

Estado del paisaje. El paisaje exhibió una tasa de deforestación del $2.6 \%$ año ${ }^{1}$ para bosque nativo primario y del $1.3 \%$ año $^{-1}$ para bosque nativo secundario, persistiendo en el 2011 el 22\% de la cobertura boscosa. Igualmente, se observó una disminución en la densidad de parches y de bordes de bosque nativo primario y secundario, incremento de la distancia entre parches, y aumento de la matriz dominada por plantaciones de especies exóticas. Según estos resultados, la «cordillera» corresponde a un estado avanzado de transformación del paisaje (Echeverría et al., 2012; McIntyre y Hobbs, 1999). En este estado del paisaje, la pérdida de fragmentos de bosques domina sobre la división de ellos y suele ocurrir posteriormente a procesos intensos de fragmentación de bosques 
Tabla 3

Vinculación entre los patrones espaciales reportados en el presente estudio y procesos ecológicos de especies amenazadas en la cordillera de Nahuelbuta.

Patrón espacial de los bosques nativos en la cordillera de Nahuelbuta reportado por el presente estudio

Pérdida de superficie. Reducción
del número de parches. Pocos
parches con área superior a
1,000 ha. $50 \%$ del área boscosa
en parches $<50$ ha. Disminución
del área del parche más grande
de bosque nativo primario

Alto índice de contraste de bosques nativos primarios y secundarios. Incremento en la complejidad de la forma de los fragmentos
Impactos ecológicos reportados por otros estudios para especies amenazadas de la cordillera de Nahuelbuta

\begin{tabular}{|c|c|}
\hline Especies & Categoría vigente \\
\hline $\begin{array}{l}\text { Lycalopex } \\
\text { culpaeus }\end{array}$ & Vulnerable \\
\hline Lycalopex griseus & $\begin{array}{l}\text { Preocupación } \\
\text { Menor }\end{array}$ \\
\hline Lycalopex fulvipes & s En Peligro \\
\hline Leopardus guigna & Vulnerable \\
\hline Puma concolor & Casi Amenazada \\
\hline Galictis cuja & Vulnerable \\
\hline Conepatus chinga & Rara \\
\hline $\begin{array}{l}\text { Campephilus } \\
\text { magellanicus }\end{array}$ & En Peligro \\
\hline
\end{tabular}

$\begin{array}{ll}\text { Aves } & - \\ & \\ & \\ \begin{array}{l}\text { Scelorchilus } \\ \text { rubecula }\end{array} & \text { Preocupación } \\ \begin{array}{l}\text { Scytalopus } \\ \text { magellanicus }\end{array} & \text { Preocupación } \\ \text { L. culpaeus } & \text { Vulnor }\end{array}$

$\begin{array}{ll}\text { L. griseus } & \text { Preocupación } \\ & \text { Menor } \\ \text { L. fulvipes } & \text { En Peligro } \\ \text { L. guigna } & \text { Vulnerable }\end{array}$
del hábitat se incrementa. Por lo tanto, RCE cambios en la estructura de la vegetación, producto de procesos de fragmentación,

RCE disminuyen la disponibilidad y uso de

RCE hábitats para las especies
RCE

Sus poblaciones están asociadas a quebradas en Rabanal y pequeños parches remanentes de bosque nativo Alarcón (2010) rodeados por plantaciones forestales de especies exóticas. Esto afecta el movimiento de anfibios en el paisaje, que dependen de hábitats conectados para el desarrollo de su ciclo vital

Telmatobufo $\quad$ Vulnerable-Rara $\mathrm{RCE}$
bullocki

Procesos de erosión relacionados con la cosecha Soto-Azat et al. y manejo de plantaciones forestales sedimentan (2012) cuerpos de agua asociados a su hábitat y cambios biofísicos, lo cual limita los recursos alimenticios y elimina las condiciones adecuadas para su reproducción

RCE Hábitats degradados, aislados y rodeados Venegas (2015) por plantaciones de especies exóticas. Baja diversidad genética, alto grado de diferenciación genética entre localidades y un elevado nivel de aislamiento, lo cual hace a la especie sensible a la fragmentación e incrementa su riesgo de extinción 
(Echeverría et al., 2012; McIntyre y Hobbs, 1999). Muy diferente fue lo observado en la India entre 1975 y 2005 (Reddy, Sreelekshmi, Jha y Dadhwal, 2013), y en el hotspot de biodiversidad al sur de Ecuador (Tapia-Armijos et al., 2015), entre 1976 y 2008, donde existió la reducción y división de grandes parches de bosque nativo, mientras, se producía un aumento en el número de parches, la longitud y densidad de borde, patrón característico de fases iniciales de fragmentación (Newton, 2007).

De continuar la trayectoria en el paisaje estudiado y careciendo de medidas en la planificación y gestión para la conservación, protección y restauración de ecosistemas boscosos, se esperaría a futuro una pérdida considerable de cobertura boscosa, con una disminución de la tasa de deforestación. Asimismo, si la tasa actual de forestación con especies exóticas se mantiene constante a la estimada $\left(4.2 \% \mathrm{año}^{-1}\right)$, es posible esperar una mayor homogeneización del paisaje con una o 2 especies de interés comercial, convirtiendo a la «cordillera» en un paisaje en estado relictual, severamente desforestado, con pequeños parches de bosque nativo altamente modificados, aislados y sin conectividad, rodeados de usos de suelo altamente contrastantes (Echeverría et al., 2012; McIntyre y Hobbs, 1999). En este estado, el ligero aumento de bosques nativos secundarios y los cambios en las trayectorias indicarían regeneración en zonas agrícolas abandonadas, tal como se ha reportado en la comuna de Ancud, región de Los Lagos, Chile (Carmona, Nahuelhual, Echeverría y Báez, 2010).

\section{Cambios en el patrón espacial de la cobertura boscosa e implicancias para la conservación de la biodiversidad}

Impactos de la disminución del tamaño de los parches hábitat. El rápido proceso de deforestación en la «cordillera» ocasionó que en tan solo 25 años se perdieran grandes fragmentos de bosque nativo primario y secundario, llegando a poseer pocos parches de tamaño superior a 1,000 ha y 50\% del área boscosa en parches menores de 50 ha. En este sentido, en el paisaje costero del río Maule-Cobquecura en Chile (al norte de la «cordillera»), se encontró que durante el periodo 1975-2000 en el primer año de estudio el $44 \%$ de la superficie boscosa se concentraba en un gran parche de entre 20,000 y 100,000 ha. Para el año 2000, el $69 \%$ de los bosques se encontraban en parches de menos de 100 ha y solo un $3 \%$ del área boscosa estaba en parches con un tamaño superior a 1,000 ha (Echeverría et al., 2006). Esta reducción en el tamaño de los parches de bosques nativos en paisajes chilenos puede afectar la disponibilidad de hábitat de diversas especies que requieren de grandes extensiones para persistir (De Angelo et al., 2011; Laurance et al., 2002; Uezu y Metzger, 2011). En este sentido, se ha reportado que especies de carnívoros presentes en la «cordillera», como el puma (Puma concolor (Linnaeus, 1771)) y el zorro de Darwin, muestran preferencias por hábitats de bosque nativo adulto y parches de gran extensión, lo cual concentraría sus poblaciones en pocos parches en la «cordillera», aumentando los niveles de superposición espacial entre las especies de carnívoros, incluido el perro asilvestrado, y la competencia por presas y territorio (MoreiraArce, Vergara y Boutin, 2015) (tabla 3). Por su parte, especies de aves amenazadas presentes en la «cordillera»y asociadas a bosques nativos primarios de interior, como el carpintero negro (Campephilus magellanicus (King, 1828)), han experimentado un declive en sus hábitats, junto con una disminución del tamaño y rango de distribución de sus poblaciones (Llabrés, 2015) (tabla 3).

Diferentes estudios realizados en diversos ecosistemas demuestran que la fragmentación y deforestación altera un conjunto de variables relacionadas con la estructura del bosque; asimismo, el tamaño de los fragmentos se relaciona significativamente con la composición de especies y la estructura de comunidades (Cadotte, Franck, Reza y Lovett-Doust, 2002; Castelletta et al., 2005; Echeverría et al., 2007; Tabarelli et al., 1999; Uezu y Metzger, 2011). Diferencias estructurales en la vegetación se han asociado con cambios en la composición de aves entre hábitats en la «cordillera» (Fontúrbel y Jiménez, 2014; Moreno-García, Zamora y Herrera, 2014) (tabla 3), respondiendo posiblemente a variables como el aumento de la irradiación y temperatura (Patten y Smith-Patten, 2012). Igualmente, se ha demostrado que la probabilidad de uso de sitio por carnívoros en riesgo de extinción, como la güiña (Leopardus guigna (Molina, 1782)), el zorro de Darwin y el puma, aumenta a medida que la diversidad estructural del hábitat se incrementa (Moreira-Arce et al., 2016) (tabla 3).

Efectos borde. En el presente estudio, los bosques nativos primario y secundario exhibieron un alto contraste debido a la interface entre usos del suelo antrópico y vegetación nativa secundaria. Estudios anteriores en Chile y el sur de Portugal reportan que las plantaciones de eucalipto (Eucalyptus globulus Labill) presentan bordes de alto contraste con el hábitat natural debido a su composición monoespecífica y estructura sencilla (Echeverría, Gatica y Fuentes, 2013; Reino et al., 2009), lo cual puede afectar directamente el movimiento de organismos (Peyras et al., 2013). En la «cordillera» se han encontrado poblaciones de anfibios en riesgo de extinción, como son el Sapo de pecho espinoso de Vanzolini y el sapo de Bullock (Telmatobufo bullocki (Schmidt, 1952)), en pequeños remanentes de bosque nativo rodeados por plantaciones de especies exóticas. Estas plantaciones afectan la movilidad de los anfibios y el desarrollo de su ciclo vital (Rabanal y Alarcón, 2010; SotoAzat, Cuevas, Flores y Valenzuela-Sánchez, 2012) (tabla 3). Igualmente, procesos de erosión relacionados con la cosecha, y manejo de plantaciones forestales, generan cambios biofísicos y la sedimentación de cuerpos de agua asociados a su hábitat, eliminando las condiciones adecuadas para su reproducción (Soto-Azat et al., 2012) (tabla 3).

Efectos de aislamiento entre hábitats. Un patrón espacial discontinuo de hábitats, como el que se describe para el área de estudio, puede conducir a una disminución de la conectividad funcional entre sitos aptos para las especies y a una alteración de la capacidad de dispersión en función de las condiciones de la matriz que las separa (Bennett, 1999; Uezu y Metzger, 2011). Esto induce, a su vez, a un incremento del riesgo de extinciones locales, al hacer más vulnerables a las especies ante procesos estocásticos, catástrofes naturales, amenazas humanas y pérdida de variabilidad genética (Bennett, 1999; Hooftman, Billeter, Schmid y Diemer, 2004; Lindenmayer y Fischer, 2006). En este sentido, se ha demostrado que poblaciones de la especie 
arbórea pitao ( $P$. punctata) en la «cordillera», y de distribución restringida en la cordillera de la costa en Chile, presentan una baja variabilidad genética dentro de cada localidad, y un alto grado de diferenciación genética entre localidades por efecto del aislamiento entre poblaciones (Venegas, 2015) (tabla 3), comparado con otras especies arbóreas del centro sur de Chile, como el queule y el hualo (Lophozonia glauca (Phil.) Heenan y Smissen.) (Delaveau et al., 2013; Vergara, Gitzendanner, Soltis y Soltis, 2014). De acuerdo con esto, se puede inferir que la $P$. punctata se encuentra en un cuello de botella genético, más aun si se considera que su área de ocupación es inferior a 1,000 ha y que en la naturaleza persisten menos de 1,000 individuos maduros (Hechenleitner et al., 2005).

La cordillera de Nahuelbuta debe ser considerada como un paisaje premium de conservación, ya que aún concentra altos niveles de biodiversidad y endemismo, pero a la vez un estado avanzado de transformación y progresiva fragmentación de los bosques nativos. Esta condición premium dentro del hotspot de biodiversidad justifica la urgencia de salvaguardar las diferentes especies de flora y fauna cuyo riesgo de extinción puede incrementarse debido a la pérdida y modificación de sus hábitats. Esta condición también se fundamenta por su bajo grado de protección $( \pm 10.4 \%)$, existiendo actualmente 10 áreas de alto valor de conservación con una superficie aproximada de 57,500 ha, siendo la quebrada Caramávida el área de conservación más extensa en el paisaje (37,000 ha). Las 2 únicas áreas resguardadas por el Sistema Nacional de Áreas Silvestres Protegidas del Estado son el Parque Nacional Nahuelbuta (6,832 ha) y el Monumento Natural Contulmo ( $82 \mathrm{ha}$ ), cuyas extensiones difícilmente pueden sostener la alta biodiversidad de la zona (Armesto, Rozzi, Smith-Ramírez y Arroyo, 1998; Simonetti y Mella, 1997).

Se sugiere la implementación de manera urgente de estrategias para la planificación de la conservación en la cordillera de Nahuelbuta que impliquen: restauración de ecosistemas degradados, conectividad de fragmentos boscosos en el paisaje, conservación de especies amenazadas en el paisaje, actualización de sitios prioritarios para la conservación de la biodiversidad, implementación de nuevas áreas protegidas y análisis de los impactos ecológicos de pérdida de hábitat en especies y comunidades de flora y fauna.

Por último, se sugiere que futuras investigaciones aborden las causas de transformación del paisaje y la pérdida de bosque nativo en la «cordillera», con el fin de ser incluidas en la toma de acciones para la conservación de la biodiversidad y planificación del territorio.

\section{Agradecimientos}

CONICYT y la «Beca Nacional de Doctorado para Extranjeros sin Residencia Definitiva». Proyecto FONDECYT 1140531 y el Laboratorio de Ecología del Paisaje por la información brindada; al Dr. Anibal Pauchad y Dra. Dolors Armenteras por sus comentarios y observaciones. Ms. C. Rodrigo Fuentes por la información y ayuda suministrada.

\section{Referencias}

Aguayo, M., Pauchard, A., Azócar, G. y Parra, O. (2009). Cambio del uso del suelo en el centro sur de Chile a fines del siglo XX. Entendiendo la dinámica espacial y temporal del paisaje. Revista Chilena de Historia Natural, 82, 361-374.

Armesto, J. J., Rozzi, R., Smith-Ramírez, C. y Arroyo, M. T. K. (1998). Conservation targets in South American temperate forests. Science, 282, 1271-1272.

Barbosa, O. y Marquet, P. (2002). Effects of forest fragmentation on the beetle assemblage at the relict forest of Fray Jorge, Chile. Oecologia, 132, 296-306.

Bennett, A. F. (1999). Linkages in the landscape: the role of corridors and connectivity in wildlife conservation. Gland, Switzerland and Cambridge, UK: IUCN.

Cadotte, M. W., Franck, R., Reza, L. y Lovett-Doust, J. (2002). Tree and shrub diversity and abundance in fragmented littoral forest of southeastern Madagascar. Biodiversity \& Conservation, 11, 1417-1436.

Carmona, A., Nahuelhual, L., Echeverría, C. y Báez, A. (2010). Linking farming systems to landscape change: an empirical and spatially explicit study in southern Chile. Agriculture, Ecosystems \& Environment, 139, 40-50.

Castelletta, M., Thiollay, J. M. y Sodhi, N. S. (2005). The effects of extreme forest fragmentation on the bird community of Singapore Island. Biological Conservation, 121, 135-155

Chander, G., Markham, B. L. y Helder, D. L. (2009). Summary of current radiometric calibration coefficients for Landsat MSS, TM, ETM+, and EO-1 ALI sensors. Remote Sensing of Environment, 113, 893-903.

CIREN (Centro de Información de Recursos Naturales). (1999). Descripciones de suelos. Materiales y símbolos. Estudio agrológico VII y IX Región. Santiago: Centro de Information de Recursos Naturales (CIREN). Publicación CIREN Núm. 121.

Corporación Nacional Forestal (Conaf), Comisión Nacional de Medio Ambiente (Conama), Banco Internacional de Reconstrucción y Fomento (Birf), Universidad Austral de Chile, Pontificia Católica de Chile y Universidad Católica de Temuco. (1999). Catastro y evaluación de los recursos vegetacionales nativos de Chile. Informe nacional con variables ambientales. Santiago: Gobierno Nacional de Chile.

Crooks, K. R., Burdett, C. L., Theobald, D. M., Rondinini, C. y Boitani, L. (2011). Global patterns of fragmentation and connectivity of mammalian carnivore habitat. Philosophical Transactions of the Royal Society B-Biological Sciences, 366, 2642-2651.

Cushman, S. A., McGarigal, K. y Neel, M. C. (2008). Parsimony in landscape metrics: strength, universality, and consistency. Ecological Indicators, 8, 691-703.

De Angelo, C., Paviolo, A. y di Bitetti, M. (2011). Differential impact of landscape transformation on pumas (Puma concolor) and jaguars (Panthera onca) in the Upper Paraná Atlantic Forest. Diversity and Distributions, 17, 422-436

Delaveau, C., Fuentes-Arce, G., Ruiz, E., Hasbún, R., Uribe, M. y Valenzuela, S. (2013). Variabilidad genética mediante AFLP en tres relictos de Gomortega keule (Molina) Baillon: especie endémica chilena en peligro de extinción. Gayana Botánica, 70, 188-194.

Di Castri, F. y Hajek, E. R. (1976). Bioclimatología de Chile. Santiago: Editorial Universidad Católica de Chile.

Echeverría, C., Coomes, D., Salas, J., Rey-Benayas, J. M., Lara, A. y Newton, A. (2006). Rapid deforestation and fragmentation of Chilean Temperate Forests. Biological Conservation, 130, 481-494.

Echeverría, C., Gatica, P. y Fuentes, R. (2013). Habitat edge contrast as an indicator to prioritize sites for ecological restoration at the landscape scale. Natureza \& Conservação, 11, 170-175.

Echeverría, C., Newton, A., Lara, A., Rey-Benayas, J. M. y Coomes, D. (2007). Impacts of forest fragmentation on species composition and forest structure in the temperate landscape of southern Chile. Global Ecology and Biogeography, 16, 426-439.

Echeverría, C., Newton, A., Nahuelhual, L., Coomes, D. y Rey-Benayas, J. M. (2012). How landscapes change: integration of spatial patterns and human processes in temperate landscapes of southern Chile. Applied Geography, $32,822-831$ 
Fontúrbel, F. E. y Jiménez, J. E. (2014). Does bird species diversity vary among forest types? A local-scale test in Southern Chile. Naturwissenschaften, 101, 855-859.

Forman, R. T. y Godron, M. (1986). Landscape ecology. New York: Wiley.

Green, J. M. H., Larrosa, C., Burgess, N. D., Balmford, A., Johnston, A., Mbilinyi, B. P., et al. (2013). Deforestation in an African biodiversity hotspot: extent, variation and the effectiveness of protected areas. Biological Conservation, 164, 62-72.

Hechenleitner, V., Gardner, M. F., Thomas, P. I., Echeverría, C., Escobar, B., Brownless, P., et al. (2005). Plantas amenazadas del centro-sur de Chile. Distribución, conservación y propagación. Chile: Universidad Austral de Chile y Real Jardín Botánico de Edimburgo.

Hooftman, D. A., Billeter, R. C., Schmid, B. y Diemer, M. (2004). Genetic effects of habitat fragmentation on common species of Swiss fen meadows. Conservation Biology, 18, 1043-1051.

Huete, A. R. (1988). A soil-adjusted vegetation index (SAVI). Remote Sensing of Environment, 25, 295-309.

IDRISI (software), (2012). Selva help system. Clark University: Clark Labs.

Lara, A., Araya, L., Capella, J., Fierro, M. y Cavieres, A. (1989). Evaluación de la destrucción y disponibilidad de los recursos forestales nativos en la VII y VIII Región. Santiago: Informe Técnico, Comité Pro Defensa Fauna y Flora.

Laurance, W. F., Lovejoy, T. E., Vasconcelos, H. L., Bruna, E. M., Didham, R. K., Stouffer, P. C., et al. (2002). Ecosystem decay of Amazonian forest fragments: a 22-year investigation. Conservation Biology, 16, 605-618.

Lindenmayer, D. B. y Fischer, J. (2006). Habitat fragmentation and landscape change: an ecological and conservation synthesis. Washington: Island Press.

Liu, J. y Taylor, W. W. (2002). Integrating landscape ecology into natural resource management. Cambridge UK: Cambridge Univ. Press.

Llabrés, V. J. (2015). Variación de los patrones espaciales del bosque nativo adulto de Nothofagus de la cordillera de Nahuelbuta y su relación con la abundancia de Campephilus magallanicus (King, 1828) (Tesis Ingeniería en Conservación de Recursos Naturales). Concepción, Chile: Facultad de Ciencias Forestales.

López-Barrera, F. (2004). Estructura y función en bordes de bosques. Revista Ecosistemas, 13, 55-68.

Luebert, F. y Pliscoff, P. (2006). Sinopsis bioclimática y vegetacional de Chile. Santiago: Editorial Universitaria.

Mardones, M. (2005). La cordillera de la Costa: caracterización físico-ambiental y regiones morfoestructurales. En C. Smith-Ramírez, J. J. Armesto, y C. Valdovinos (Eds.), Historia, biodiversidad y ecología de los bosques costeros de Chile (pp. 39-59). Santiago, Chile: Editorial Universitaria.

Mas, J. F., Gao, Y. y Pacheco, J. A. N. (2010). Sensitivity of landscape pattern metrics to classification approaches. Forest Ecology and Management, 259, $1215-1224$.

McGarigal, K., Cushman, S., Neel, M. y Ene, E. (2013). FRAGSTATS. Spatial pattern analysis program for categorical maps. University of Massachusetts (Landscape Ecology Program) [consultado 7Ago 2013]. Disponible en: http://www.umass.edu/landeco/research/fragstats/fragstats.html

McIntyre, S. y Hobbs, R. (1999). A framework for conceptualizing human effects on landscapes and its relevance to management and research models. Conservation Biology, 13, 1282-1292.

Miranda, A., Altamirano, A., Cayuela, L., Lara, A. y González, M. (2017). Native forest loss in the Chilean biodiversity hotspot: revealing the evidence. Regional Environmental Change, 17, 285-297.

Mittermeier, R. A., Turner, W. R., Larsen, F. W., Brooks, T. M. y Gascon, C. (2011). Global biodiversity conservation: the critical role of hotspots. En F. E. Zachos y J. C. Habel (Eds.), Biodiversity hotspots: distribution and protection of conservation priority areas (pp. 3-23). Berlin, Heidelberg: Springer.

Moreira-Arce, D., Vergara, P. M. y Boutin, S. (2015). Diurnal human activity and introduced species affect occurrence of carnivores in a human-dominated landscape. Plos One, 10, e0137854.

Moreira-Arce, D., Vergara, P. M., Boutin, S., Carrasco, G., Briones, R., Soto, G. E., et al. (2016). Mesocarnivores respond to fine-grain habitat structure in a mosaic landscape comprised by commercial forest plantations in southern Chile. Forest Ecology and Management, 369, 135-143.
Moreno-García, R. A., Zamora, R. y Herrera, M. A. (2014). Habitat selection of endemic birds in temperate forests in a biodiversity "Hotspot". Forest Systems, 23, 216-224.

Newton, A. C. (2007). Biodiversity loss and conservation in fragmented forest landscapes: the forests of montane Mexico and temperate South America. Wallingford, Oxford UK: CABI.

Patten, M. A. y Smith-Patten, B. D. (2012). Testing the microclimate hypothesis: light environment and population trends of Neotropical birds. Biological Conservation, 155, 85-93.

Peng, J., Wang, Y., Zhang, Y., Wu, J., Li, W. y Li, Y. (2010). Evaluating the effectiveness of landscape metrics in quantifying spatial patterns. Ecological Indicators, 10, 217-223.

Peyras, M., Vespa, N. I., Bellocq, M. I. y Zurita, G. A. (2013). Quantifying edge effects: the role of habitat contrast and species specialization. Journal of Insect Conservation, 17, 807-820.

Rabanal, F. E. y Alarcón, D. (2010). Amphibia, Anura, Cycloramphidae, Alsodes vanzolinii (Donoso-Barros, 1974): rediscovery in nature, latitudinal and altitudinal extension in Nahuelbuta Range, southern Chile. Check List, 6, 362-363.

Reddy, C. S., Sreelekshmi, S., Jha, C. y Dadhwal, V. (2013). National assessment of forest fragmentation in India: landscape indices as measures of the effects of fragmentation and forest cover change. Ecological Engineering, 60, 453-464.

Reese, H. y Olsson, H. (2011). C-correction of optical satellite data over alpine vegetation areas: a comparison of sampling strategies for determining the empirical c-parameter. Remote Sensing of Environment, 115, 1387-1400.

Reino, L., Beja, P., Osborne, P. E., Morgado, R., Fabião, A. y Rotenberry, J. T. (2009). Distance to edges, edge contrast and landscape fragmentation: interactions affecting farmland birds around forest plantations. Biological Conservation, 142, 824-838.

Rouse, J., Jr., Haas, R., Schell, J. y Deering, D. (1974). Monitoring vegetation systems in the Great Plains with ERTS-1. In Earth Resources Technology Satellite-1, Third ERTS-1 Symposium. Washington: NASA.

SERNAGEOMIN (Servicio Nacional de Geología y Minería). (2003). Mapa geológico de Chile. Publicación Geológica Digital, Núm. 4 (CD-ROM, versión1.0). Santiago: Servicio Nacional de Geología y Minería.

Simonetti, J. y Mella, J. E. (1997). Park size and the conservation of Chilean mammals. Revista Chilena de Historia Natural, 70, 213-220.

Smith-Ramírez, C. (2004). The Chilean coastal range: a vanishing center of biodiversity and endemism in South American temperate rainforests. Biodiversity \& Conservation, 13, 373-393.

Soto-Azat, C., Cuevas, C., Flores, E. y Valenzuela-Sánchez, A. (2012). Conservación de Telmatobufo bullocki (sapo de Bullock) y su hábitat en los bosques degradados de Nahuelbuta. En C. Soto-Azat y A. Valenzuela-Sánches (Eds.), Conservación de anfibios de Chile. Memorias del Taller de Conservación de Anfibios para Organismos Públicos (pp. 70-75). Santiago: Universidad Nacional Andrés Bello.

Steininger, M., Tucker, C. J., Ersts, P., Killeen, T., Villegas, Z. y Hecht, S. (2001). Clearance and fragmentation of tropical deciduous forest in the Tierras Bajas, Santa Cruz, Bolivia. Conservation Biology, 15, 856-866.

Stratford, J. A. y Stouffer, P. C. (2015). Forest fragmentation alters microhabitat availability for Neotropical terrestrial insectivorous birds. Biological Conservation, 188, 109-115.

Tabarelli, M., Mantovani, W. y Peres, C. A. (1999). Effects of habitat fragmentation on plant guild structure in the montane Atlantic forest of southeastern Brazil. Biological Conservation, 91, 119-127.

Tapia-Armijos, M. F., Homeier, J., Espinosa, C. I., Leuschner, C. y de la Cruz, M. (2015). Deforestation and forest fragmentation in South Ecuador since the 1970s - losing a hotspot of biodiversity. Plos One, 10, e0133701.

Tucker, C. J. (1979). Red and photographic infrared linear combinations for monitoring vegetation. Remote Sensing of Environment, 8, $127-150$

Turner, I. M. (1996). Species loss in fragments of tropical rain forest: a review of the evidence. Journal of Applied Ecology, 33, 200-209.

Uezu, A. y Metzger, J. P. (2011). Vanishing bird species in the Atlantic Forest: relative importance of landscape configuration, forest structure and species characteristics. Biodiversity and Conservation, 20, 3627-3643. 
Van Laake, P. E. y Sánchez-Azofeifa, G. A. (2004). Focus on deforestation: zooming in on hot spots in highly fragmented ecosystems in Costa Rica. Agriculture, Ecosystems \& Environment, 102, 3-15.

Venegas, C. F. (2015). Variabilidad genética en subpoblaciones de Pitavia punctata Mol. Especie endémica amenazada del sur de Chile (Tesis Ingeniería en Biotecnología Vegetal). Concepción, Chile: Facultad de Ciencias Forestales, Universidad de Concepción.

Vergara, R., Gitzendanner, M. A., Soltis, D. E. y Soltis, P. S. (2014). Population genetic structure, genetic diversity, and natural history of the South American species of Nothofagus subgenus Lophozonia (Nothofagaceae) inferred from nuclear microsatellite data. Ecology and Evolution, 4, 2450-2471.

Wiegand, T., Revilla, E. y Moloney, K. A. (2005). Effects of habitat loss and fragmentation on population dynamics. Conservation Biology, 19, 108-121.

Wolodarsky-Franke, A. y Díaz, S. (2011). Cordillera de Nahuelbuta. Reserva mundial de biodiversidad. Valdivia: WWF.

Zeng, H. y Wu, X. B. (2005). Utilities of edge-based metrics for studying landscape fragmentation. Computers, Environment and Urban Systems, 29, $159-178$. 